\title{
UNIFORM PROPERTY (K) AND ITS RELATED PROPERTIES
}

\author{
Hong-Kun Xu and GiUseppe Marino
}

\begin{abstract}
A Banach space $X$ is said to have uniform property $(K)$ if there exists a constant $k \in[0,1)$ such that whenever $x_{n} \rightarrow 0,\left\|x_{n}\right\| \rightarrow 1$, and $\limsup _{m \rightarrow \infty} \limsup _{n \rightarrow \infty}\left\|x_{n}-y_{m}\right\| \leqslant$ 1 we have limsup $\left\|y_{m}\right\| \leqslant k$. This property is the uniform version of property $(K)$ recently introduced by B. Sims (Bull. Austral. Math. Soc. 50(1994), 523-528). Sufficient conditions for uniform property $(K)$ are given. Some examples are presented to separate various Banach space properties. Applications to nonlinear operators are also included.
\end{abstract}

\section{INTRODUCTION}

A Banach space $X$ is said to have weak normal structure if every weakly compact convex subset $C$ of $X$ with more than one point contains a non-diametral point, that is, an $x \in C$ for which

$$
\sup \{\|x-y\|: y \in C\}<\operatorname{diam}(C) .
$$

A characterisation for weak normal structure is that there exists no non-constant sequence $\left\{x_{n}\right\}$ in $X$ satisfying

$$
x_{n} \rightarrow 0
$$

and

$$
\lim _{n}\left\|x_{n}-x\right\|=\operatorname{diam}\left\{x_{n}\right\}=1 \text { for all } x \in \overline{\mathrm{co}}\left\{x_{n}\right\} .
$$

Here and throughout the paper, " $\rightarrow$ " stands for weak convergence and " $\rightarrow$ " for strong convergence.

Having observed the above characterisation of weak normal structure, Sims [15] introduced the following property $(K)$ which implies weak normal structure.

Received 14th April, 1997

This work was done while the the first author was visiting Dipartimento di Matematica, Universitá della Calabria, Cosenza, Italy. He would like to thank them for their hospitality. He also would like to thank Professor Brailey Sims of the University of Newcastle, Australia for stimulating discussions on property $(K)$ and other topics. Thanks also go to Professor E. Llorens-Fuster of Universitat de Valéncia, Spain for faxing us a copy of the paper [16]. The research of the first author was in part supported by the Foundation for Research Development (South Africa).

Copyright Clearance Centre, Inc. Serial-fee code: 0004-9729/98 $\$ A 2.00+0.00$. 
Definition 1: [15] A Banach space $X$ is said to have property $(K)$ if there exists a constant $k \in[0,1)$ such that whenever $x_{n} \rightarrow 0,\left\|x_{n}\right\| \rightarrow 1$, and $\lim \sup \left\|x_{n}-x\right\| \leqslant 1$ we have $\|x\| \leqslant k$.

Clearly property $(K)$ with $k=0$ is equivalent to Opial's property [14]:

$$
x_{n} \rightarrow 0 \Longrightarrow \limsup \left\|x_{n}\right\|<\lim \sup \left\|x_{n}-x\right\| \text { for all } x \in X, x \neq 0 \text {. }
$$

Property $(K)$ implies property $(P)$ introduced in [17] (but not conversely; see Example 2 in Section 3):

$$
x_{n} \rightarrow x \text { and }\left\{x_{n}\right\} \text { is non-constant } \Longrightarrow \liminf \left\|x_{n}-x\right\|<\operatorname{diam}\left\{x_{n}\right\}_{n=1}^{\infty}
$$

as property $(P)$ is equivalent to the statement below:

$$
x_{n} \rightarrow 0 \text { and }\left\|x_{n}\right\| \rightarrow 1 \Longrightarrow \operatorname{diam}\left\{x_{n}\right\}_{n=1}^{\infty}>1 .
$$

Let now $C$ be a weakly compact convex subset of a Banach space $X$ and $T: C \rightarrow C$ be an asymptotically nonexpansive mapping in the weak sense [8]; that is, $T^{N}$ is continuous for some integer $N \geqslant 1$ and such that

$$
\limsup _{n \rightarrow \infty} \sup \left\{\left\|T^{n} x-T^{n} y\right\|-\|x-y\|: y \in C\right\} \leqslant 0 \quad \forall x \in C .
$$

Recall that the norm of $X$ is UKK (uniformly Kadec-Klee) if for any $\varepsilon>0$ there exists a $\delta>0$ such that for any sequence $\left\{x_{n}\right\} \subset B_{X}$ (the closed unit ball) which is $\varepsilon$-separated (that is, $\operatorname{sep}\left(x_{n}\right):=\inf \left\{\left\|x_{n}-x_{m}\right\|: n \neq m\right\} \geqslant \varepsilon$ ), the weak convergence of $\left\{x_{n}\right\}$ to $x$ implies that $\|x\| \leqslant 1-\delta$. This UKK property implies property $(K)$ [15]. Lin [11] recently proved that if the norm of $X$ is UKK and $x \in C$ is such that $T^{n} x \rightarrow x$, then $x$ is a fixed point of $T$. However in an attempt to weaken Lin's UKK assumption, we could not recover the same result if UKK is replaced by Sims' property $(K)$. A stronger property than property $(K)$ is needed. This motivated us to propose the following notion of uniform property $(K)$.

Definition 2: A Banach space $X$ is said to have uniform property $(K)$ if there exists a constant $k \in[0,1)$ such that whenever $x_{n} \rightarrow 0,\left\|x_{n}\right\| \rightarrow 1$, and $\underset{m \rightarrow \infty}{\limsup } \limsup _{n \rightarrow \infty}\left\|x_{n}-y_{m}\right\| \leqslant 1$ we have $\limsup _{m \rightarrow \infty}\left\|y_{m}\right\| \leqslant k$.

Clearly uniform property $(K)$ implies property $(K)$. It is also clear that uniform property $(K)$ with $k=0$ is exactly the locally uniform Opial property [12]; namely, $x_{n} \rightarrow x$ and $\limsup _{m} \limsup _{n}\left\|x_{n}-y_{m}\right\| \leqslant \limsup _{n}\left\|x_{n}-x\right\| \Rightarrow y_{m} \rightarrow x$.

The paper is organised as follows. After the notion of uniform property $(K)$ in the Introduction, we give certain conditions in Section 2 which are sufficient for the uniform property $(K)$. In Section 3 we present a few examples of Banach spaces which distinguish various Banach space properties. Finally in Section 4 we are concerned with the demiclosedness principle, asymptotic behaviour, and existence of fixed points of asymptotically nonexpansive mappings in the weak sense. 


\section{Sufficient Conditions for Uniform Property $(K)$}

Throughout the rest of this paper we always assume that a Banach space $X$ is not Schur; that is, $X$ has a weakly convergent sequence which is not strongly convergent.

Recall that Opial's modulus of a Banach space $X$ is defined by [12]

$$
r_{X}(c):=\inf \left\{\liminf \left\|x_{n}+x\right\|-1\right\},
$$

where the infimum is taken over all sequences $\left\{x_{n}\right\}$ such that $x_{n} \rightarrow 0$ and $\left\|x_{n}\right\| \rightarrow 1$ and all $x \in X$ such that $\|x\| \geqslant c . \quad X$ is said to have the uniform Opial property if $r_{X}(c)>0$ for all $c>0$. The number

$$
c_{0}(X):=\sup \left\{c>0: r_{X}(c) \leqslant 0\right\}
$$

is called the Opial characteristic of $X$.

Proposition 1. If $c_{0}(X)<1$, then $X$ has uniform property $(K)$ with $k=$ $c_{0}(X)$.

Proof: Assume we have $x_{n} \rightarrow 0,\left\|x_{n}\right\| \rightarrow 1$, and $\underset{m}{\limsup } \limsup _{n}\left\|x_{n}-y_{m}\right\| \leqslant 1$. It follows from the definition of Opial's modulus that

$$
1 \geqslant \underset{m}{\limsup } \limsup _{n}\left\|x_{n}-y_{m}\right\| \geqslant 1+r_{X}\left(\lim \sup \left\|y_{m}\right\|\right)
$$

since $r_{X}(\cdot)$ is continuous [12]. Thus $r_{X}\left(\limsup \left\|y_{m}\right\|\right) \leqslant 0$ and limsup $\left\|y_{m}\right\| \leqslant$ $c_{0}(X)$.

A Banach space $X$ is called $\varepsilon_{0}$-UKK if, for some $\varepsilon_{0} \in(0,1)$, there exists $\delta>0$ such that whenever $\left\{x_{n}\right\} \subset B_{X}$ (the closed unit ball of $\left.X\right), x_{n} \rightarrow x$ and $\operatorname{sep}\left(x_{n}\right):=$ $\inf \left\{\left\|x_{n}-x_{m}\right\|: n \neq m\right\} \geqslant \varepsilon_{0}$, it follows that $\|x\| \leqslant 1-\delta$.

Proposition 2. If $X$ is $\varepsilon_{0}$-UKK for some $\varepsilon_{0} \in(0,1)$, then $X$ satisfies uniform property $(K)$.

Proof: Suppose $x_{n} \rightarrow 0,\left\|x_{n}\right\| \rightarrow 1$, and limsup limsup $\left\|x_{n}-y_{m}\right\| \leqslant 1$. Let $\delta>0$ be the number given in the definition of $\varepsilon_{0}$-UKK. Let $\eta>0$ be small enough so that $(1+\eta) \varepsilon_{0}<1$. As in the proof of Sims [15, Proposition 5] we can select a subsequence of $\left\{x_{n}\right\}$, still denoted $\left\{x_{n}\right\}$, such that $\operatorname{sep}\left(x_{n}\right)>(1+\eta) \varepsilon_{0}$. Choose now an integer $N$ large enough so that

$$
\underset{n}{\limsup }\left\|x_{n}-y_{m}\right\|<1+\eta \quad \forall m \geqslant N .
$$

For a fixed $m \geqslant N$, then choose an $n_{0}=n_{0}(m, \eta)$ such that

$$
\left\|x_{n}-y_{m}\right\|<1+\eta \quad \forall n \geqslant n_{0} .
$$


Then we have

$$
\frac{x_{n}-y_{m}}{1+\eta} \rightarrow \frac{-y_{m}}{1+\eta} \quad \text { and } \quad \operatorname{sep}\left(\frac{x_{n}-y_{m}}{1+\eta}\right) \geqslant \varepsilon_{0} .
$$

It follows from $\varepsilon_{0}-\mathrm{UKK}$ that

$$
\left\|y_{m}\right\| \leqslant(1+\eta)(1-\delta) \quad \forall m \geqslant N
$$

so

$$
\lim \sup \left\|y_{m}\right\| \leqslant 1-\delta
$$

A Banach space $X$ is said to be $\varepsilon_{0}$-inquadrate if $\delta_{X}\left(\varepsilon_{0}\right)>0$, where $\delta_{X}(\cdot)$ is the modulus of convexity of $X$ defined by

$$
\delta_{X}(\varepsilon)=\inf \left\{1-\left\|\frac{x+y}{2}\right\|:\|x\| \leqslant 1,\|y\| \leqslant 1,\|x-y\| \geqslant \varepsilon\right\} .
$$

Also a Banach space $X$ is said to satisfy the non-strict Opial property if $x_{n} \rightarrow x$ implies that $\lim \sup \left\|x_{n}-x\right\| \leqslant \lim \sup \left\|x_{n}-y\right\|$ for all $y \in X$.

Proposition 3. If $X$ is $\varepsilon_{0}$-inquadrate for some $\varepsilon_{0} \in(0,1)$ and satisfies the non-strict Opial property, then $X$ has the uniform property $(K)$ with $k=\varepsilon_{0}$.

PRoof: Assume that $x_{n} \rightarrow 0,\left\|x_{n}\right\| \rightarrow 1$, and limsuplimsup $\left\|x_{n}-y_{m}\right\| \leqslant 1$. Put $d=\limsup \left\|y_{m}\right\|$. If $d=0$, there is nothing to prove; so assume $d>0$. For $\eta>0$ take $N$ so large that

$$
\underset{n}{\limsup }\left\|x_{n}-y_{m}\right\|<1+\eta \quad \forall m \geqslant N .
$$

For a fixed $m \geqslant N$, take $n_{0}=n_{0}(m, \eta)$ such that

$$
\left\|x_{n}\right\|<1+\eta \quad \text { and } \quad\left\|x_{n}-y_{m}\right\|<1+\eta \quad \forall n \geqslant n_{0}
$$

It follows that

$$
\left\|x_{n}-\frac{1}{2} y_{m}\right\|=\left\|\frac{x_{n}+\left(x_{n}-y_{m}\right)}{2}\right\| \leqslant(1+\eta)\left[1-\delta_{X}\left(\frac{\left\|y_{m}\right\|}{1+\eta}\right)\right] .
$$

The non-strict Opial property then implies that for $m \geqslant N$,

$$
1=\lim \left\|x_{n}\right\| \leqslant \limsup \left\|x_{n}-\frac{1}{2} y_{m}\right\| \leqslant(1+\eta)\left[1-\delta_{X}\left(\frac{\left\|y_{m}\right\|}{1+\eta}\right)\right],
$$

which in turn implies

$$
\delta_{X}(d) \leqslant 0
$$


and therefore $\lim \sup \left\|y_{m}\right\| \leqslant \varepsilon_{0}$.

A Banach space $X$ is said to have weak orthogonality (WORTH) [15] if $x_{n} \rightarrow 0$ implies that

$$
\left\|x_{n}+x\right\|-\left\|x_{n}-x\right\| \rightarrow 0 \quad \forall x \in X
$$

Proposition 4. If $X$ is $\varepsilon_{0}$-inquadrate with $\varepsilon_{0} \in(0,2)$ (that is, $X$ is uniformly non-square) and WORTH, then $X$ has the uniform property ( $K$ ) with $k=\varepsilon_{0} / 2$.

Proof: Given $x_{n} \rightarrow 0,\left\|x_{n}\right\| \rightarrow 1$, and limsup limsup $\left\|x_{n}-y_{m}\right\| \leqslant 1$. Then for any $\eta>0$, we can choose $N$ such that

$$
\underset{n}{\limsup }\left\|x_{n}-y_{m}\right\|<1+\frac{1}{2} \eta \quad \forall m \geqslant N .
$$

Next for a fixed $m \geqslant N$, choose $n_{0}=n_{0}(m, \eta)$ such that for all $n \geqslant n_{0}$ we have

$$
\begin{aligned}
\left\|x_{n}-y_{m}\right\| & <1+\frac{1}{2} \eta, \\
\left\|x_{n}\right\| & >1-\eta, \\
\left|\left\|x_{n}+y_{m}\right\|-\left\|x_{n}-y_{m}\right\|\right| & <\frac{1}{2} \eta .
\end{aligned}
$$

It follows that$$
1-\eta<\left\|x_{n}\right\|=\left\|\frac{1}{2}\left[\left(x_{n}-y_{m}\right)+\left(x_{n}+y_{m}\right)\right]\right\|
$$$$
\leqslant(1+\eta)\left[1-\delta_{X}\left(\frac{2\left\|y_{m}\right\|}{1+\eta}\right)\right] \text {. }
$$

This immediately implies that

$$
\delta_{X}\left(2 \limsup \left\|y_{m}\right\|\right) \leqslant 0 .
$$

Hence

$$
\lim \sup \left\|y_{m}\right\| \leqslant \frac{1}{2} \varepsilon_{0}
$$

A Banach space $X$ is said to have the generalised Gossez-Lami Dozo property (GGLD for short) [7] whenever

$$
D\left[\left(x_{n}\right)\right]>1
$$

for all $x_{n} \rightarrow 0$ such that $\left\|x_{n}\right\| \rightarrow 1$, where

$$
D\left[\left(x_{n}\right)\right]=\underset{m}{\limsup } \limsup _{n}\left\|x_{n}-x_{m}\right\| .
$$

Proposition 5. Uniform property $(K)$ with $k \in[0,1)$ implies GGLD.

Proof: Suppose $X$ has the uniform property $(K)$ with $k \in[0,1)$. Assume $x_{n} \rightarrow$ 0 , $\left\|x_{n}\right\| \rightarrow 1$. If $D\left[\left(x_{n}\right)\right] \leqslant 1$, then the uniform property $(K)$ implies that

$$
1=\lim \left\|x_{m}\right\| \leqslant k<1,
$$

a contradiction. 


\section{Some EXAmples}

Sims' property $(K)$ with $k=0$ is exactly Opial's property, but uniform property $(K)$ with $k \in(0,1)$ does not necessarily imply Opial's property.

EXAMPLE 1. Let $X=l^{2} \oplus \mathbb{R}$ be normed by

$$
\|(x, r)\|=\|x\|_{2}+\max \left\{|r|-\frac{1}{2}\|x\|_{2}, 0\right\}, \quad(x, r) \in X,
$$

where $\|\cdot\|_{2}$ is the usual $l^{2}$ norm. Let $\left\{e_{n}\right\}$ be the unit basis of $l^{2}$. Considering the sequence $y_{n}=\left(e_{n}, 0\right)$ and the point $y=(0, \alpha)$ with $|\alpha| \leqslant 1 / 2$, we see that $X$ fails to have Opial's property. (It is not hard to see $X$ does have the non-strict Opial property.) However, since it has been calculated in [18] that

$$
r_{X}(c)=\frac{c+\sqrt{1+c^{2}}}{2}-1, \quad c>0
$$

it follows that $c_{0}(X) \leqslant 3 / 4$ and thus $X$ satisfies the uniform property $(K)$ by Proposition 1.

Recall that a Banach space $X$ is said to have uniform normal structure [1] if the normal structure coefficient of $X$ defined by

$$
N(X)=\inf \left\{\operatorname{diam}(K) / r_{K}(K)\right\}
$$

is bigger than one, where the infimum is taken over all closed bounded convex subsets $K$ of $X$ consisting of more than one point, $\operatorname{diam}(K)=\sup \{\|x-y\|: x, y \in K\}$ is the diameter of $K$ and $r_{K}(K)=\inf _{x \in K} \sup _{y \in K}\|x-y\|$ is the self-Chebyshev radius of $K$. It is shown [13] that uniform normal structure implies reflexivity. Sims' property $(K)$ implies weak normal structure, however even uniform normal structure does not imply property $(K)$ as the following example shows.

EXAMPLE 2. Let $X=l^{2} \oplus \mathbb{R}$ with the norm

$$
\|(x, r)\|=\max \left\{\|x\|_{2},|r|\right\} .
$$

Then $N(X)=\sqrt{2}[2]$ and hence $X$ has uniform normal structure. However, considering the sequence $x_{n}=\left(e_{n}, 0\right)$ and the point $x=(0,1)$, we immediately find that $X$ lacks property $(K)$ for all $k \in[0,1)$. This example also shows that property $(P)$ does not imply property $(K)$, in general.

Propery $(K)$ does not imply uniform property $(K)$; in fact, the next example shows that even Opial's property does not implies GGLD. 
EXAMPLE 3. Let $c_{0}$ be renormed by

$$
\|x\|=\sqrt{\|x\|_{\infty}^{2}+\sum_{i=1}^{\infty} \frac{1}{2^{i}} x_{i}^{2}}, \quad x=\left(x_{n}\right) \in c_{0},
$$

where $\|\cdot\|_{\infty}$ is the usual $l_{\infty}$ norm. It is known [5] that $\left(c_{0},\|\cdot\|\right)$ is UCED (uniformly convex in every direction) but lacks GGLD. In order to show that $\left(c_{0},\|\cdot\|\right)$ satisfies Opial's property, according to Sims [15, Proposition 1], we need only to show that $\left(c_{0},\|\cdot\|\right)$ satisfies the non-strict Opial property:

$$
x_{n} \rightarrow 0,\left\|x_{n}\right\| \rightarrow 1 \quad \Longrightarrow \quad \liminf \left\|x_{n}-x\right\| \geqslant 1 \quad \forall x \neq 0
$$

Write $x_{n}=\left(\xi_{i}^{n}\right)$ and $x=\left(\xi_{i}\right)$. Take $i_{0}$ such that $\left|\xi_{i_{0}}\right|=\|x\|_{\infty}$. For $\varepsilon>0$ there exists $i_{1}>i_{0}$ large enough so that

$$
\left|\xi_{i}\right|<\varepsilon \quad \forall i \geqslant i_{1} .
$$

As $x_{n} \rightarrow 0$, we have $\xi_{i}^{n} \rightarrow 0($ as $n \rightarrow \infty)$ for each $i$. Select $N>i_{1}$ such that

$$
\left|\xi_{i}^{n}\right|<\varepsilon \quad \forall n \geqslant N, \quad 1 \leqslant i \leqslant i_{1} .
$$

Thus for $n \geqslant N$,

$$
\max _{1 \leqslant i \leqslant i_{1}}\left|\xi_{i}^{n}-\xi_{i}\right| \geqslant \max _{1 \leqslant i \leqslant i_{1}}\left(\left|\xi_{i}\right|-\left|\xi_{i}^{n}\right|\right) \geqslant\|x\|_{\infty}-\varepsilon
$$

and

$$
\sup _{i>i_{1}}\left|\xi_{i}^{n}-\xi_{i}\right| \geqslant \sup _{i>i_{1}}\left(\left|\xi_{i}^{n}\right|-\left|\xi_{i}\right|\right) \geqslant\left\|x_{n}\right\|_{\infty}-\varepsilon \quad \forall n \geqslant N .
$$

It follows that

$$
\left\|x_{n}-x\right\|_{\infty} \geqslant \max \left\{\left\|x_{n}\right\|_{\infty},\|x\|_{\infty}\right\}-\varepsilon \quad \forall n \geqslant N
$$

and

$$
\lim \inf \left\|x_{n}-x\right\|_{\infty} \geqslant \max \left\{\|x\|_{\infty}, 1\right\}
$$

since $x_{n} \rightarrow 0$ implies $\lim \left\|x_{n}\right\|_{\infty}=\lim \left\|x_{n}\right\|=1$. Now we have

$$
\begin{aligned}
\liminf \left\|x_{n}-x\right\|^{2} & =\liminf \left(\left\|x_{n}-x\right\|_{\infty}^{2}+\sum_{i=1}^{\infty} \frac{\left(\xi_{i}^{n}-\xi_{i}\right)^{2}}{2^{i}}\right) \\
& \geqslant \max \left\{\|x\|_{\infty}^{2}, 1\right\}+\sum_{i=1}^{\infty} \frac{\left(\xi_{i}\right)^{2}}{2^{i}} \\
& \geqslant \max \left\{\|x\|^{2}, 1\right\} \geqslant 1 .
\end{aligned}
$$


Since property $(K)$ implies weak normal structure, while uniform property $(K)$ is the uniform version of property $(K)$, one might conjecture that uniform property $(K)$ would imply the weak uniform normal structure, the uniform version of weak normal structure. However, unfortunately, the answer is negative as the example below shows. But first let us recall that a Banach space $X$ is said to have weak uniform normal structure if Bynum's weakly convergent coefficient $W C S(X)$ is bigger than one, where

$$
W C S(X)=\inf \left\{\operatorname{diam}(K) / r_{K}(K)\right\}
$$

the infimum being taken over all weakly compact convex subsets $K$ of $X$ with more than one point.

EXAMPLE 4. Let

$$
X=\left(\sum_{i=2}^{\infty} \oplus l^{i}\right)_{l^{2}} .
$$

It is known [16] that $X$ has GGLD. It is also known [3] that $X$ is UCED. As it is easily seen that $X$ has a basis $\left\{e_{n}\right\}_{n=1}^{\infty}$ such that $\left\|I-Q_{n}\right\|=1$ for all $n \geqslant 1$, where $I$ is the identity and $Q_{n}$ is the natural projection associated with the basis $\left\{e_{n}\right\}$; that is, $Q_{n}(x)=\sum_{i=1}^{n} a_{i} e_{i}$ if $x=\sum_{i=1}^{\infty} a_{i} e_{i}$, it follows from van Dulst [4] that $X$ satisfies the nonstrict Opial property. (This together with Proposition 1 of Sims [15] further implies that $X$ indeed satisfies Opial's property.) Now we show that $X$ enjoy the uniform property ( $K$ ) with $k=0$ (that is, $X$ satisfies the locally uniform Opial property). Towards this end, we assume $\left\{x^{n}\right\}$ is a sequence in $X$ such that $x^{n} \rightarrow 0$ and $\left\|x^{n}\right\| \rightarrow 1$. Assume $\left\{y^{m}\right\}$ is any sequence of $X$ such that

$$
\limsup _{m} \limsup _{n}\left\|x^{n}-y^{m}\right\| \leqslant 1
$$

Let $P_{i}$ denote the natural projection onto the subspace of $X$ identified with $l^{i}$ for $i>1$. First we show that

$$
\lim _{m}\left\|P_{i}\left(y^{m}\right)\right\|=0 \quad \forall i>1
$$

Suppose $\underset{m}{\limsup }\left\|P_{i}\left(y^{m}\right)\right\|>0$ for some $i>1$. Without loss of generality we may assume that there exist the limits

$$
a_{i}:=\lim _{n}\left\|P_{i}\left(x^{n}\right)\right\| \quad \text { and } \quad b_{i}:=\lim _{n}\left\|\left(I-P_{i}\right)\left(x^{n}\right)\right\| .
$$

We may also assume that there exists the $\lim _{m}\left\|P_{i}\left(y^{m}\right)\right\|=: c_{i}$. (Note: $c_{i}>0$.) As $\left\|x^{n}\right\| \rightarrow 1$, we see that

$$
a_{i}^{2}+b_{i}^{2}=1
$$


Recall the fact (see [9]) that in any $l^{p}(1<p<\infty)$ if $\left\{u_{n}\right\}$ converges weakly to $u \in l^{p}$, then $\liminf \left\|u_{n}-v\right\|^{p}=\liminf \left\|u_{n}-u\right\|^{p}+\|v-u\|^{p}$ for all $v \in l^{p}$. It follows from the Opial property that

$1 \geqslant \underset{m}{\limsup } \limsup _{n}\left\|x^{n}-y^{m}\right\|^{2}$

$=\underset{m}{\limsup } \limsup _{n}\left(\left\|P_{i}\left(x^{n}\right)-P_{i}\left(y^{m}\right)\right\|^{2}+\left\|\left(I-P_{i}\right)\left(x^{n}\right)-\left(I-P_{i}\right)\left(y^{m}\right)\right\|^{2}\right)$

$\geqslant \underset{m}{\limsup }\left(\liminf _{n}\left\|P_{i}\left(x^{n}\right)-P_{i}\left(y^{m}\right)\right\|^{2}+\liminf _{n}\left\|\left(I-P_{i}\right)\left(x^{n}\right)-\left(I-P_{i}\right)\left(y^{m}\right)\right\|^{2}\right)$

$\geqslant \underset{m}{\limsup }\left[\left(\liminf _{n}\left\|P_{i}\left(x^{n}\right)\right\|^{i}+\left\|P_{i}\left(y^{m}\right)\right\|^{i}\right)^{2 / i}+\liminf _{n}\left\|\left(I-P_{i}\right)\left(x^{n}\right)\right\|^{2}\right]$

$=\left(a_{i}^{i}+c_{i}^{i}\right)^{2 / i}+b_{i}^{2}$

$>a_{i}^{2}+b_{i}^{2}=1$.

This contradiction proves the claim $(*)$.

Set

$$
\lambda_{n}:=\left\{\left\|P_{i}\left(x^{n}\right)\right\|\right\}_{i \geqslant 2} \quad \text { and } \quad \mu_{m}:=\left\{\left\|P_{i}\left(y^{m}\right)\right\|\right\}_{i \geqslant 2} .
$$

Then $\left\{\lambda_{n}\right\} \subset l^{2}$ and $\left\|\lambda_{n}\right\| \rightarrow 1$, and $\left\{\mu_{m}\right\} \subset l^{2}$ and $\mu_{m} \rightarrow 0$ in $l^{2}$ (This follows from (*).) We may assume that $\left\{\lambda_{n}\right\}$ converges weakly to some $\lambda \in l^{2}$. It follows that

$$
\begin{aligned}
& 1 \geqslant \lim \sup \lim \sup \left\|x^{n}-y^{m}\right\|^{2} \text {. } \\
& m \text { n } \\
& =\underset{m}{\limsup } \limsup _{n} \sum_{i=2}^{\infty}\left\|P_{i}\left(x^{n}\right)-P_{i}\left(y^{m}\right)\right\|^{2} \\
& \geqslant \underset{m}{\limsup } \limsup _{n} \sum_{i=2}^{\infty}\left|\left\|P_{i}\left(x^{n}\right)\right\|-\left\|P_{i}\left(y^{m}\right)\right\|\right|^{2} \\
& =\limsup _{m} \underset{n}{\lim \sup }\left\|\lambda_{n}-\mu_{m}\right\|_{l^{2}}^{2} \\
& =\underset{m}{\limsup } \limsup _{n}\left(\left\|\lambda_{n}\right\|_{l^{2}}^{2}-2\left\langle\lambda_{n}, \mu_{m}\right\rangle+\left\|\mu_{m}\right\|_{l^{2}}^{2}\right) \\
& =\underset{m}{\limsup }\left(1-2\left\langle\lambda, \mu_{m}\right\rangle+\left\|\mu_{m}\right\|_{l^{2}}^{2}\right) \\
& =1+\underset{m}{\limsup }\left\|\mu_{m}\right\|_{l^{2}}^{2} \text {. }
\end{aligned}
$$

Therefore,

$$
\underset{m}{\limsup }\left\|y^{m}\right\|^{2}=\underset{m}{\limsup }\left\|\mu_{m}\right\|_{l^{2}}^{2} \leqslant 0 .
$$

This finishes the proof. 
REMARK 1. Example 4 also presents a Banach space $X$ which satisfies the locally uniform Opial property but fails to satisfy the uniform Opial property as for any $c>0$, $r_{X}(c) \leqslant r_{l^{i}}(c)=\left(1+c^{i}\right)^{1 / i}-1([12]) \rightarrow 0$ as $i \rightarrow \infty$.

The following is an example of a Banach space which has Opial's property and GGLD but lacks the locally uniform Opial property.

EXAMPLE 5. Let

$$
X=\left(l^{2} \oplus l^{3}\right)_{\infty}
$$

with the norm

$$
|z|:=\|z\|_{\infty}+\sum_{i=1}^{\infty} \frac{\left|x_{i}\right|+\left|y_{i}\right|}{2^{i}}
$$

where $z=(x, y) \in X$ with $x=\left(x_{i}\right) \in l^{2}$ and $y=\left(y_{i}\right) \in l^{3}$, and $\|z\|_{\infty}=\|x\|_{2} \vee\|y\|_{3}$, $\|\cdot\|_{p}$ being the usual $l^{p}$ norm. (Here $a \vee b=\max \{a, b\}$ for real numbers $a$ and $b$.)

We now show that this space $X$ does enjoy Opial's property and GGLD but lacks the locally uniform Opial property. Assume $z^{n}=\left(x^{n}, y^{n}\right) \rightarrow 0$ and $\left|z^{n}\right| \rightarrow 1$. We may also assume (otherwise select a subsequence if necessary) that there exist the limits: $a:=\lim \left\|x^{n}\right\|_{2}$ and $b:=\lim \left\|y^{n}\right\|_{3}$. Since $\left|z^{n}\right| \rightarrow 1$, it readily follows that $a \vee b=1$. We now claim that

$$
\text { (**) } \quad \lim \left|z^{n}-z\right|=\left(a^{2}+\|x\|_{2}^{2}\right)^{1 / 2} \vee\left(b^{3}+\|y\|_{3}^{3}\right)^{1 / 3}+\sum_{i=1}^{\infty} \frac{\left|x_{i}\right|+\left|y_{i}\right|}{2^{i}}
$$

for any $z=(x, y) \in X$ with $x=\left(x_{i}\right) \in l^{2}$ and $y=\left(y_{i}\right) \in l^{3}$. Again we use the identity in $l^{p}(1<p<\infty)$ (see $\left.[9]\right)$ :

$$
\left\{u^{n}\right\} \subset l^{p}, u_{n} \rightarrow 0 \Rightarrow \liminf \left\|u^{n}-v\right\|_{p}^{p}=\liminf \left\|u^{n}\right\|_{p}^{p}+\|v\|_{p}^{p} \quad \forall v \in l^{p} .
$$

(Note: The same identity holds if "lim inf" is replaced by "limsup".)

It then follows that

$$
\begin{aligned}
\lim \left|z^{n}-z\right| & =\lim \left(\left\|z^{n}-z\right\|_{\infty}+\sum_{i=1}^{\infty} \frac{\left|x_{i}^{n}-x_{i}\right|+\left|y_{i}^{n}-y_{i}\right|}{2^{i}}\right) \\
& =\lim \left(\left\|x^{n}-x\right\|_{2} \vee\left\|y^{n}-y\right\|_{3}\right)+\sum_{i=1}^{\infty} \frac{\left|x_{i}\right|+\left|y_{i}\right|}{2^{i}} \\
& =\left(a^{2}+\|x\|_{2}^{2}\right)^{1 / 2} \vee\left(b^{3}+\|y\|_{3}^{3}\right)^{1 / 3}+\sum_{i=1}^{\infty} \frac{\left|x_{i}\right|+\left|y_{i}\right|}{2^{i}}
\end{aligned}
$$

which is the claim $(* *)$. In order to show the Opial property we assume $z=(x, y) \neq 0$. Then it follows from $(* *)$ that in either case $a=1$ or $b=1$ we have

$$
\lim \sup \left|z_{n}-z\right| \geqslant 1+\sum_{i=1}^{\infty} \frac{\left|x_{i}\right|+\left|y_{i}\right|}{2^{i}}>1 \text {. }
$$


So $X$ does satisfy the Opial property. Next we turn to the GGLD. If $a=1$ we have

$$
\lim _{n}\left\|x^{n}-x^{m}\right\|_{2}=\sqrt{1+\left\|x^{m}\right\|_{2}^{2}} \rightarrow \sqrt{2} \quad \text { as } m \rightarrow \infty .
$$

If $b=1$ we have

$$
\lim _{n}\left\|y^{n}-y^{m}\right\|_{3}=\sqrt[3]{1+\left\|y^{m}\right\|_{3}^{3}} \rightarrow \sqrt[3]{2} \quad \text { as } m \rightarrow \infty .
$$

So again from the claim $(* *)$ it follows that

$$
\begin{aligned}
& \limsup \limsup \left|z^{n}-z^{m}\right| \\
& m \\
& \begin{array}{l}
\geqslant \limsup _{m}\left\{\left(a^{2}+\left\|x^{m}\right\|_{2}^{2}\right)^{1 / 2} \vee\left(b^{3}+\left\|y^{m}\right\|_{3}^{3}\right)^{1 / 3}+\sum_{i=1}^{\infty} \frac{\left|x_{i}^{m}\right|+\left|y_{i}^{m}\right|}{2^{i}}\right\} \\
=\sqrt{2} a \vee \sqrt[3]{2} b \geqslant \sqrt[3]{2}>1 \quad \text { since } a \vee b=1
\end{array}
\end{aligned}
$$

So $X$ has GGLD. Finally we show that $X$ does not satisfy the locally uniform Opial property. Take any $z^{n}=\left(x^{n}, y^{n}\right) \rightarrow 0$ with $\left|z^{n}\right| \rightarrow 1$ such that $\left\|x^{n}\right\|_{2} \rightarrow 1$ while $\left\|y^{n}\right\|_{3} \rightarrow b<1$. Choose another sequence $w^{n}=\left(u^{n}, v^{n}\right)$ such that $\left\|u^{m}\right\|_{2} \rightarrow 0$, $\left\|v^{m}\right\|_{3} \rightarrow c>0$ such that $b^{3}+c^{3} \leqslant 1$, and $v_{i}^{m} \rightarrow 0$ as $m \rightarrow \infty$ for each $i \geqslant 1$. Then once again from the claim $(* *)$ it follows that

$$
\begin{aligned}
\underset{m}{\limsup } \limsup _{n}\left|z^{n}-w^{m}\right| & =\underset{m}{\limsup }\left(\sqrt{1+\left\|u^{m}\right\|_{2}^{2}} \vee \sqrt[3]{b^{3}+\left\|v^{m}\right\|_{3}^{3}}+\sum_{i=1}^{\infty} \frac{\left|u_{i}^{m}\right|+\left|v_{i}^{m}\right|}{2^{i}}\right) \\
& =1 \vee \sqrt[3]{b^{3}+c^{3}}=1 .
\end{aligned}
$$

This verifies that $X$ lacks the locally uniform Opial property as $\left\{w^{m}\right\}$ does not converge strongly to 0 .

REMARK 2. The above proof to the GGLD in fact shows that $\operatorname{WCS}(X)=\sqrt[3]{2}$ and hence $X$ has the weak uniform normal structure.

\section{Asymptotically Nonexpansive Mappings in the Weak Sense}

Let $C$ be a weakly compact convex subset of a Banach space $X$ and $T: C \rightarrow C$ be an asymptotically nonexpansive mapping in the weak sense (see the Introduction for definition). Set

$$
\varepsilon_{n}(x):=\max \left\{\sup \left(\left\|T^{n} x-T^{n} y\right\|-\|x-y\|: y \in C\right), 0\right\} .
$$

Then

$$
\lim _{n} \varepsilon_{n}(x)=0 \quad \forall x \in C
$$


and

$$
\left\|T^{n} x-T^{n} y\right\| \leqslant\|x-y\|+\varepsilon_{n}(x) \quad \forall x, y \in C \quad \forall n \geqslant 1 .
$$

We say that $I-T$ is demiclosed (at 0 ) provided

$$
x_{n} \rightarrow x \text { and }(I-T) x_{n} \rightarrow 0 \quad \Longrightarrow \quad(I-T) x=0 .
$$

In [18] (see also [12]) it is proved that if $X$ has the locally uniform Opial property, then $I-T$ is demiclosed. The following strictly (see Example 5 in Section 3 ) extends this result.

THEOREM 1. If $X$ satisfies Opial's property and has GGLD, then $I-T$ is demiclosed (at 0 ).

Proof: Assume $x_{n} \rightarrow x$ and $(I-T) x_{n} \rightarrow 0$. From [18, Lemma 4.1] (see also [12, Lemma 3.1]) it follows that $T^{n} x \rightarrow x$. This convergence must be strong; for otherwise, GGLD implies

$$
\begin{aligned}
\underset{n}{\limsup \left\|T^{n} x-x\right\|} & <\underset{m}{\limsup } \underset{n}{\limsup }\left\|T^{n} x-T^{m} x\right\| \\
& \leqslant \limsup _{m} \limsup _{n}\left(\left\|T^{n-m} x-x\right\|+\varepsilon_{m}(x)\right) \\
& =\underset{n}{\limsup }\left\|T^{n} x-x\right\| .
\end{aligned}
$$

This is a contradiction. Therefore, $T^{n} x \rightarrow x$ and $x$ is a fixed point of $T$ since $T^{N}$ is continuous for some $N \geqslant 1$.

REMARK 3. If $T$ is uniformly continuous, then the conclusion of Theorem 1 is proved in $[6]$.

The next two results show that the UKK assumption in the main theorem of [11] and respectively, the assumption that $D(X)<1$ (or equivalently $W C S(X)>1$ ) in [10, Theorem 4] can strictly be both weakened to GGLD.

THEOREM 2. If $X$ satisfies Opial's property and GGLD, then for a given $x \in C$, $\left\{T^{n} x\right\}$ converges weakly to a fixed point of $T$ if and only if $T$ is weakly asymptotically regular at $x$; that is, $w-\lim _{n}\left(T^{n} x-T^{n+1} x\right)=0$.

Proof: We need only show the sufficiency part; so assume $T^{n} x-T^{n+1} x \rightarrow 0$. Let $y=w-\lim _{i} T^{n_{i}} x$ be any weak cluster point of $\left\{T^{n} x\right\}$. We need to prove that $y$ is a fixed point of $T$. As $T$ is weakly asymptotically regular at $x$, we have

$$
w-\lim _{i} T^{n_{i}+m} x=y \quad \forall m \geqslant 0 .
$$

Put

$$
c_{m}=\max \left\{\sup \left(\left\|T^{m} z-T^{m} y\right\|-\|z-y\|: z \in C\right), 0\right\}
$$


and

$$
b_{m}=\limsup _{i}\left\|T^{n_{i}+m} x-y\right\| .
$$

From Opial's property it follows that

$$
\begin{aligned}
b_{m+j} & =\limsup _{i}\left\|T^{n_{i}+m+j} x-y\right\| \\
& \leqslant \limsup _{i}\left\|T^{n_{i}+m+j} x-T^{j} y\right\| \\
& \leqslant \limsup _{i}\left(\left\|T^{n_{i}+m} x-y\right\|+c_{j}\right) \\
& =b_{m}+c_{j} .
\end{aligned}
$$

So $\lim \sup b_{j} \leqslant b_{m}$ for all $m \geqslant 0$. Hence

$j$

$$
b:=\lim _{m} b_{m}=\inf \left\{b_{m}: m \geqslant 0\right\}
$$

If $b=0$, then

$$
\begin{aligned}
\underset{j}{\limsup }\left\|T^{j} y-y\right\| & \leqslant \underset{j}{\limsup } \underset{m}{\limsup \lim \sup }\left(\left\|T^{j} y-T^{n_{i}+m+j} x\right\|+\left\|T^{n_{i}+m+j} x-y\right\|\right) \\
& \leqslant \underset{j}{\lim \sup } \underset{m}{\lim \sup }\left(b_{m}+c_{j}+b_{m+j}\right) \\
& =0 .
\end{aligned}
$$

So $y$ is a fixed point of $T$. If $b>0$, we may assume without loss of generality that $b=1$. Then Lin [11] has constructed a subsequence $\left\{m_{j}\right\}$ with $m_{j} \in\left\{n_{i}+l: i, l \in \mathbb{N}\right\}$ such that

(i) $T^{m_{j}} x \rightarrow y$;

(ii) $1-(1 / j) \leqslant\left\|T^{m_{j}} x-y\right\| \leqslant 1+(1 / j)$;

(iii) $\left\|T^{m_{j}} x-T^{i} y\right\| \leqslant 1+c_{i}+(1 / j) \quad \forall 0<i \leqslant j$.

Consequently, if $z$ is a weak limit point of $\left\{T^{i} y\right\}$, then

$$
\begin{aligned}
\underset{j}{\limsup }\left\|T^{m_{j}} x-z\right\| & \leqslant \underset{i}{\limsup } \underset{j}{\lim \sup }\left\|T^{m_{j}} x-T^{i} y\right\| \\
& \leqslant 1=\underset{j}{\limsup }\left\|T^{m_{j}} x-y\right\| .
\end{aligned}
$$

Opial's property then gives that $z=y$. Thus we conclude that $T^{i} y \rightarrow y$, from which GGLD entails that $T^{i} y \rightarrow y$ and hence $y$ is a fixed point of $T$. To complete the proof we have to show that if $u=w-\lim _{j} T^{r_{j} x}$ is also a weak limit point of $\left\{T^{n} x\right\}$, then we must have $u=y$. Indeed, if $u \neq y$, then noting that $\lim \left\|T^{n} x-p\right\|$ exists for 
every fixed point $p$ of $T$ and that $u$ is also a fixed point of $T$, we deduce from Opial's property that

$$
\begin{aligned}
\lim _{n}\left\|T^{n} x-y\right\| & =\lim _{i}\left\|T^{n_{i}} x-y\right\| \\
& <\lim _{i}\left\|T^{n_{i}} x-u\right\|=\lim _{j}\left\|T^{r_{j}} x-u\right\| \\
& <\lim _{j}\left\|T^{r_{j}} x-y\right\|=\lim _{n}\left\|T^{n} x-y\right\| .
\end{aligned}
$$

This is a contradiction.

REMARK 4. In [6] the same conclusion as stated in Theorem 2 is proved under the stronger condition that $T$ is asymptotically regular at $x$; that is, $\lim \left\|T^{n+1} x-T^{n} x\right\|=$ 0 .

THEOREM 3. If $X$ has GGLD and if $T$ is, in addition, weakly asymptotically regular on $C$ (that is, $w-\lim \left(T^{n} x-T^{n+1} x\right)=0$ for all $x \in C$ ), then $T$ has a fixed point.

Proof: First as in the proof of [10, Theorem 4], there exists a subsequence $\left\{n_{i}\right\}$ of $\mathbb{N}$ and a point $x \in C$ such that $T^{n_{i}} x \rightarrow x$. We may further assume that $n_{i}-n_{j}>i-j$ for all $i>j$ and $\lim \left\|T^{n_{i}} x-x\right\|$ exists. Since $T$ is weakly asymptotically regular at $x$ we have $w-\lim _{i} T^{n_{i}+m} x=x$ for all $m \geqslant 0$. From the weak lower semicontinuity of the norm it follows that for $i>j$,

$$
\begin{aligned}
\left\|T^{n_{i}} x-T^{n_{j}} x\right\| & \leqslant\left\|T^{n_{i}-n_{j}} x-x\right\|+\varepsilon_{n_{j}}(x) \\
& \leqslant \liminf _{s}\left\|T^{n_{s}+\left(n_{i}-n_{j}\right)} x-T^{n_{i}-n_{j}} x\right\|+\varepsilon_{n_{j}}(x) \\
& \leqslant \liminf _{s}\left\|T^{n_{s}} x-x\right\|+\varepsilon_{n_{i}-n_{j}}(x)+\varepsilon_{n_{j}}(x) .
\end{aligned}
$$

Hence

$$
\underset{j}{\limsup } \limsup _{i}\left\|T^{n_{i}} x-T^{n_{j}} x\right\| \leqslant \lim \left\|T^{n_{s}} x-x\right\| .
$$

GGLD then implies that $\lim \left\|T^{n_{s}} x-x\right\|=0$. As $T^{N}$ is continuous and $T$ is weakly asymptotically regular at $x$, it follows that $T^{N} x=x$. Now we have

$$
T x-x=T^{i N+1} x-T^{i N} x \rightarrow 0 \quad \text { as } i \rightarrow \infty .
$$

Therefore $T x=x$.

\section{REFERENCES}

[1] W.L. Bynum, 'Normal structure coefficients for Banach spaces', Pacific J. Math. 86 (1980), 427-436. 
[2] E. Casini and E. Maluta, 'Fixed points of uniformly Lipschitzian mappings in spaces with uniformly normal structure', Nonlinear Anal. 9 (1985), 103-106.

[3] M.M. Day, R.C. James and S. Swaminathan, 'Normed linear spaces that are uniformly convex in every direction', Canad. J. Math. 23 (1971), 1051-1059.

[4] D. van Dulst, 'Equivalent norms and the fixed point property for nonexpansive mappings', J. London Math. Soc. 25 (1982), 139-144.

[5] J. Garciá-Falset and E. Lloréns-Fuster, 'Normal structure and fixed point property', Glasgow Math. J. 38 (1996), 29-37.

[6] J. Garcia-Falset, B. Sims and M.A. Smyth, 'The demiclosedness principle for mappings of asymptotically nonexpansive type', Houston J. Math. 22 (1996), 101-108.

[7] A. Jiménez-Melado, 'Stability of weak normal structure in James quasi reflexive space', Bull. Austral. Math. Soc. 46 (1992), 367-372.

[8] W.A. Kirk, 'Fixed point theorems for non-Lipschitzian mappings of asymptotically nonexpansive type', Israel J. Math. 17 (1974), 339-346.

[9] T.C. Lim, 'Asymptotic centers and nonexpansive mappings in some conjugate spaces', Pacific J. Math. 90 (1980), 135-143.

[10] T.C. Lim and H.K. Xu, 'Fixed point theorems for asymptotically nonexpansive mappings', Nonlinear Anal. 22 (1994), 1345-1355.

[11] P.K. Lin, 'Asymptotic behavior for asymptotically nonexpansive mappings', Nonlinear Anal. 26 (1996), 1137-1141.

[12] P.K. Lin, K.K. Tan and H.K. Xu, 'Demiclosedness principle and asymptotic behavior for asymptotically nonexpansive mappings', Nonlinear Anal. 24 (1995), 929-946.

[13] E. Maluta, 'Uniformly normal structure and related coefficients', Pacific J. Math. 111 (1984), 357-369.

[14] Z. Opial, 'Weak convergence of the sequence of successive approximations for nonexpasive mappings', Bull. Amer. Math. Soc. 73 (1967), 591-597.

[15] B. Sims, 'A class of spaces with weak normal structure', Bull. Austral. Math. Soc. 50 (1994), 523-528.

[16] B. Sims and M. Smyth, 'Non-uniform conditions giving weak normal structure', Quaestiones Math. 18 (1995), 9-19.

[17] K.K. Tan and H.K. Xu, 'On fixed point theorems of nonexpansive mappings in product spaces', Proc. Amer. Math. Soc. 113 (1991), 983-989.

[18] H.K. Xu, 'Geometrical coefficients of Banach spaces and nonlinear mappings', in Recent advances on metric fixed point theory, ( $\mathrm{T}$. Dominguez Benavides, Editor), Proceedings of the International Workshop on Metric Fixed Point Theory, Sevilla, Spain, September 1995 (University of Sevilla Press, 1996), pp. 161-178.

Department of Mathematics University of Durban-Westville

Durban 4000

South Africa

e-mail: hkxu@pixie.udw.ac.za
Dipartimento di Matematica

Universitá della Calabria

Cosenza

Italy

e-mail: gmarino@ccuws4.unical.it 